\title{
Informe forense: imputabilidad y trastorno antisocial de la personalidad.
}

\author{
Forensic report: imputability and antisocial personality \\ disorder.
}

\section{HA. Pallaro' y D. González-Trijueque ${ }^{2}$}

\begin{abstract}
RESUMEN
La psicología forense constituye una especialización clásica en países anglosajones y que en España está tomando cada día mayor relevancia, siendo habitual que los peritos asesoren a nuestros tribunales y jueces en materias de carácter psicológico. En el presente artículo se aborda de manera conjunta por un médico forense y un psicólogo forense un supuesto habitual en la práctica pericial, como es la valoración de la imputabilidad de un sujeto que presenta un trastorno antisocial de la personalidad. Asimismo, se realiza una introducción al concepto legal de imputabilidad y se facilita un modelo de informe emitido al respecto, complementando los resultados obtenidos con diverso apoyo bibliográfico.
\end{abstract}

Palabras clave: informe forense, imputabilidad, trastorno antisocial de la personalidad, psicopatía.

\section{ABSTRACT}

The forensic psychology constitutes a classic specialization in Anglo-Saxon countries and that in Spain is taking every major day relevancy, being habitual that the experts advise our courts and judges in matters of psychological character. In the present article a habitual supposition is approached in a joint way by a forensic doctor and a forensic psychologist in the expert practice, since it is the valuation of the imputability of a subject that presents an antisocial disorder of the personality. Likewise, an introduction is realized to the legal concept of imputability and a model of report is facilitated expressed in the matter, complementing the results obtained with diverse bibliographical support.

Key words: forensic report, imputability, antisocial personality disorder, psychopathy.

Cuad Med Forense 2009; 15(55):55-66

${ }^{1}$ Médico forense. Juzgados de Getafe.

2 Psicólogo forense. Tribunal Superior de Justicia de Madrid. 


\section{INTRODUCCIÓN:}

Aunque la imputabilidad es un concepto estrictamente jurídico [I], la valoración de la misma constituye una materia a medio camino entre el mundo del derecho y el ámbito científico ya que la repercusión de las anomalías o alteraciones psíquicas en la ejecución de hechos delictivos y en la responsabilidad criminal de su autor se encuentra recogida en el artículo 20. I de nuestro vigente Código Penal, tratándose de una materia jurídica pero claramente impregnada de un evidente carácter multidisciplinar [2].

La existencia de trastornos mentales en los sujetos que delinquen ha sido una realidad constante en la historia del ser humano, no obstante, la valoración jurídico-penal que dichos supuestos han merecido no siempre han sido resueltos del mismo modo; de hecho, los tribunales de justicia han de enfrentarse ante supuestos en los que han de pronunciarse sobre la imputabilidad de un sujeto que padece una anomalía o alteración psicopatológica, por ello los profesionales del derecho se han esforzado en poner a su disposición a la ciencia para documentar sus resoluciones doctrinales como jurisprudenciales, pretendiéndose dotar de un carácter científico a la dogmática penal [2].

El concepto de imputabilidad proviene del latín imputare que significa atribuir, configurando uno de los constructos más controvertidos que conforman la teoría jurídica del delito, siendo un concepto que puede ser abordado desde diversos posicionamientos dogmáticos, prácticos, médicopsiquiátricos y psicológicos $[1,2]$. No obstante, la base de la conducta que interesa a efectos punitivos es la culpabilidad, que posee dos elementos integrantes: un elemento de hecho, de contenido antropológico, la imputabilidad, y otro de derecho de contenido filosófico-jurídico, la responsabilidad [3].

Aunque el concepto de imputabilidad no se encuentra definido en nuestro Código Penal, podemos admitir que, en general "será considerado totalmente imputable todo aquel mayor de edad penal que no presente anomalía, alteración mental, intoxicación o abstinencia a drogas psicotrópicas, alteraciones de la percepción o trastorno mental transitorio (no buscado de propósito para delinquir) en el momento de los hechos que afecte la comprensión de lo ilícito (capacidad cognoscitiva) o la capacidad para adecuar su conducta a dicha comprensión (capacidad volitiva)" [3]. De hecho, la condición de imputable se presume en todo ser humano y su afectación deberá ser probada como el hecho delictivo mismo, según doctrina reiterada del Tribunal Supremo [2,3].

Además, la fórmula española constituye una fórmula mixta, bio/psicopatológica-psicológica (STS 12-3-1996), es decir, exige una base patológica (alteración, anomalía, etc.) y un efecto psicológico (alteración de la conciencia o voluntad) [I,3]. Esto quiere decir que no es suficiente con que exista y se pruebe la existencia de un trastorno mental, sino que es imprescindible demostrar que tal entidad nosológica ha afectado significativamente la capacidad del sujeto para comprender la ilicitud del acto realizado o poder haber actuado de una forma diferente [3].

En el presente artículo se aborda de manera conjunta por un médico forense y un psicólogo forense un supuesto habitual en la práctica pericial, como es la valoración de la imputabilidad de un sujeto que presenta un trastorno antisocial de la personalidad. Cabe señalar al respecto que desde los distintos colegios oficiales de psicólogos se recoge la figura profesional del psicólogo jurídico desde hace años y que más recientemente, desde la Consejería de Justicia e Interior de la Comunidad de Madrid se señaló que el psicólogo forense, en dependencia funcional del órgano judicial al que está adscrito, es el profesional competente y responsable de la emisión de informes periciales propios de su especialidad y que como experto, asesora a los Tribunales acerca del funcionamiento y aspectos psicológicos de las personas implicadas en los procesos judiciales de quien se solicite el correspondiente informe [4]. 
Los psicólogos forenses han accedido a las pericias sobre imputabilidad fundamentalmente por la expansión tanto legal como jurisprudencial de las causas que eximen o atenúan la responsabilidad criminal, de hecho, la consideración de que los trastornos de la personalidad, algunos estados psicológicos, los trastornos perceptivos, las anomalías o alteraciones psíquicas, etc., pueden modificar la condición de imputable obliga en muchos casos a recurrir al peritaje de expertos psicólogos ya que pueden operar desde una óptica dimensional más operativa a las necesidades jurídicas que el tradicional, aunque ya también superado, criterio categorial médico-psiquiátrico [3].

En el caso expuesto a continuación se valora un supuesto habitual de la práctica pericial referente a la imputabilidad en un sujeto que presenta un trastorno de la personalidad. Debemos señalar al respecto y con carácter introductorio que durante un tiempo se aludió de un modo más o menos genérico bajo la denominación de "personalidades psicopáticas" [5,6] a todos los trastornos de la personalidad, de hecho, en la jurisprudencia de nuestro país, bajo la denominación de psicopatía se han venido englobando muy diversas alteraciones de la personalidad, no solamente los trastornos antisociales $[1,7]$. No obstante, el término psicopatía se ha visto sujeto a numerosas disputas y conflictos por la confusión terminológica existente ya que el problema está en que los términos usados para referirse a esta anomalía o patrón conductual, hacen referencia a dos áreas distintas: I) la conducta, y 2) los rasgos de personalidad [6].

A lo largo de la historia, esta diferenciación se ha ido manteniendo, a través de la convivencia entre dos tradiciones o escuelas:

- Por un lado la tradición angloamericana iniciada con Prichard en 1835 y su concepto de "locura moral". Según esta escuela en determinadas personas se producía una pérdida de la capacidad para orientarse según los principios morales [I]. La "locura moral" sería la causa de la desviación social, mientras que la causa del trastorno se ponía en la conducta desviada, es decir se pretendía describir la psicopatía en términos conductuales y de desviación social. Esta misma perspectiva es recogida en los distintos manuales diagnósticos DSM que se han referido a esta patología con nombres como trastorno sociopático de personalidad, reacción antisocial, hasta el actual trastorno antisocial de la personalidad, el cual está definido por la presencia de conductas antisociales persistentes por lo que, las características de personalidad del sujeto no son esenciales para el diagnostico $[1,6]$.

- Por otra parte, la tradición alemana, define la psicopatía según sus rasgos de personalidad como una desviación personal, siendo el punto de referencia Koch [6]. Aunque puede que exista inadaptación a las normas sociales, lo primordial no son las conductas antisociales, sino los rasgos de personalidad que subyacen a éstas. Mientras que las clasificaciones DSM han sido más próximas a la tradición angloamericana, la clasificación de la Organización Mundial de la Salud en su última versión CIE-I0 define el trastorno disocial de la personalidad en la línea de la tradición germánica sobre psicopatía, ya que los criterios que definen el trastorno se refieren exclusivamente a características de personalidad $[6,8]$.

A pesar de los esfuerzos de distintos autores, continua la polémica sobre esta patología ya que se plantea la pregunta de si los términos usados por las distintas escuelas aluden a un único concepto unitario o si en realidad estamos hablando de entidades distintas [6].

Por último, respecto al trastorno de la personalidad que presenta el sujeto peritado en el modelo de informe facilitado a continuación, debemos señalar que los rasgos de personalidad son patrones persistentes de formas de percibir, relacionarse y pensar sobre el entorno y sobre uno 
mismo que se ponen de manifiesto en una amplia gama de contextos sociales y personales; no obstante, los rasgos de personalidad sólo constituyen trastornos de la personalidad cuando son inflexibles y desadaptativos y cuando causan un deterioro funcional significativo o un malestar subjetivo [9]. En el caso del informe aportado, el tipo de trastorno de la personalidad detectado se corresponde con el de tipo antisocial, trastorno caracterizado por un patrón general de desprecio y violación de los derechos de los demás, que comienza en la infancia o el principio de la adolescencia y continúa en la edad adulta [9].

\section{BREVE DESCRIPCIÓN DE LOS HECHOS: (1)}

Según documentación examinada (atestado $n^{\circ}$ xxx instruido desde comisaría de Parla), el 5 de Mayo de 2005 comparecieron funcionarios del cuerpo de Policía Local haciendo entrega de un gato elevador de vehículos, un destornillador y un cuchillo de $19 \mathrm{~cm}$. de longitud, presentando en calidad de detenido al Sr. JGV y dando cuenta sobre hechos acaecidos a las 2.00 horas de la madrugada en un establecimiento comercial destinado a la venta de productos tecnológicos y de electrónica, ya que recibieron una llamada telefónica mediante la que se comunicaba que en el lugar de los hechos se encontraba un joven que había roto el cristal del escaparate y había accedido al interior del mismo. El Sr. JGV no ofreció resistencia al momento de su detención.

Posteriormente, el I de Noviembre de 2005 mediante escrito del Ministerio Fiscal se señaló que los hechos referenciados eran constitutivos de un delito de robo con fuerza en grado de tentativa.

\section{OBJETO DEL INFORME:}

Se interesa por SSa que "de forma conjunta por el psicólogo forense y el médico forense adscritos a estos Juzgados se examine al acusado a fin de que se emita informe sobre la estructura de personalidad de base del mismo y en que medida ésta pueda afectar a sus capacidades cognoscitivas y volitivas en relación a los hechos denunciados", todo ello en relación al procedimiento abreviado xxx/2006 seguido en el Juzgado de lo Penal correspondiente.

\section{METODOLOGÍA:}

- Entrevista clínica semiestructurada con el Sr. JGV, realizada el I6 de Mayo de 2008.

- Administración del "Listado de Psicopatía Revisado" (Psychopathy Check-List Revised) (PCL-R), realizada el 16 de Mayo de 2008. El PCL-R es una escala de 20 ítems diseñados para medir conductas y rasgos de personalidad relacionados con la psicopatía. La prueba se completa a partir de una entrevista semiestructurada y la revisión de toda la documentación que sea relevante. La escala se compone de 2 factores principales, el factor I se centra en los rasgos afectivos interpersonales, mientras que el factor 2 comprende aspectos conductuales $[\mathrm{I} 0, \mathrm{I} \mathrm{I}]$.

- Administración del "Inventario Clínico Multiaxial de Millon" (Millon Clinical Multiaxial Inventory) (MCMI-II), realizada el 16 de Mayo de 2008. El MCMI-II es un instrumento psicométrico adaptado en población española compuesto por 175 ítems que evalúan las siguientes dimensiones: I) fiabilidad y validez del estilo de respuesta, 2) personalidad, 3) personalidad patológica, 4) síndromes clínicos de gravedad moderada, y 5) síndromes clínicos de gravedad severa [12].

(1) Los datos facilitados han sido modificados para impedir el reconocimiento del caso. 
- Administración del "Inventario Multifásico de Personalidad de Minnesota" (Minnesota Multiphasic Personaliy Inventory) (MMPI-2), realizada el 30 de Mayo de 2008. El MMPI-2 es un instrumento psicométrico adaptado en población española que permite la evaluación de la personalidad normal y patológica, con la posibilidad de valorar hasta 77 variables diferentes, contando con 3 escalas de validez, 10 escalas básicas, 15 escalas de contenido, 18 escalas suplementarias y 3 I subescalas [13].

- Vaciado de autos: análisis de la documentación que consta en el expediente judicial del caso.

\section{APROXIMACIÓN PSICOBIOGRÁFICA:}

El Sr. JGV nace en Madrid el 3 de Marzo de 1985, refiriendo una infancia y adolescencia plenamente inestables y conflictivas debido a los problemas de drogadicción de su figura materna y a su estancia en distintos internados y reformatorios ("me escapé muchas veces de esos sitios porque no me gustaban"). Informa sobre la ausencia de figuras de referencia durante su desarrollo psicoevolutivo así como de vínculos afectivos significativos, describiendo haber sido criado con importante marginación afectiva y con estilos educativos de tipo punitivo/aversivo en los distintos centros en los que permaneció. Durante su infancia y adolescencia el peritado describe déficit significativo en el establecimiento de relaciones interpersonales normalizadas, reconociendo carencias en sus habilidades sociales que contribuyeron a configurar su estructura de personalidad.

Respecto a su trayectoria delictiva, el explorado reconoce haber realizado numerosos robos y atracos desde los diecisiete años de edad ("antes de ser mayor de edad ya robaba coches y tiendas, a partir de los diecinueve años empecé a hacer atracos con colegas con armas de fuego y también alunizajes, he atracado en gasolineras, hoteles y prostíbulos [...] siempre he usado armas blancas y a veces armas de fuego, me gusta intimidar a la gente y sentir que harán lo que les pida [...] creo que el sistema es injusto y no debería ser delito robar a los ricos"), actos delictivos siempre orientados, según sus propias palabras, a conseguir dinero efectivo o artículos que le resultasen especialmente atractivos para mantener su nivel de vida ("con el dinero que sacaba era capaz de vivir mejor que mucha gente trabajando doce horas al día"). Manifiesta que desde verano de 2005 se encuentra preso por primera vez, refiriendo una buena adaptación al entorno penitenciario.

A nivel familiar, el explorado refiere tener tres hijos ("cada hijo lo he tenido con una chica distinta, la verdad es que no sé nada de ellos porque cuando rompía la relación perdía el contacto con sus madres"), describiendo una importante promiscuidad sexual desde temprana edad. Asimismo, informa no haber conocido a su padre, añadiendo que su figura materna nunca le facilitó información al respecto ("no sé nada de él, ni quien es, tampoco quiero saberlo"). Además, señala que su madre continua presentando problemas de drogadicción, no sabiendo su paradero ("la verdad es que no sé donde está, tampoco me importa mucho, no me gusta la gente débil y ella lo es"). Además, informa ser el menor de un total de tres hermanos con los que apenas mantiene relación ("mi hermano mayor también tuvo problemas con las drogas y con la justicia"). Por otra parte, el explorado refiere una inexistente relación con los demás miembros de su entorno familiar, añadiendo que a nivel social ha mantenido abundantes relaciones superficiales y poco duraderas con personas relacionadas con distintas actividades ilegales y de los que considera haberse aprovechado ("creo que nunca he tenido amigos") y que a nivel sentimental nunca ha gozado de relaciones estables ("he tenido muchas parejas pero creo que nunca me he enamorado"), no mostrando ningún tipo de vínculo emocional hacia sus familiares ni hacia otras personas de referencia. 
El Sr. JGV refiere haber cursado hasta $2^{\circ}$ de la ESO, refiriendo abundantes conflictos interpersonales, tanto con profesores como con compañeros, e historia de absentismo al respecto. A nivel laboral, señala haber desarrollado únicamente trabajos esporádicos y de corta duración en el sector de hostelería como camarero ("nunca he sido responsable con nada, tampoco me ha hecho falta mantener los trabajos porque si cometía algún robo me daba cuenta de que podía vivir bien sin trabajar"), trabajos que nunca ha mantenido ya sea por su consumo de sustancias o por su estilo de vida delictivo.

El reconocido no informa sobre antecedentes psicopatológicos familiares de relevancia ni sobre tratamientos psicofarmacológicos o psicoterapéuticos al respecto, a excepción de los problemas de drogadicción ya mencionados. A nivel personal, el Sr. JGV no refiere psicopatología conocida de interés ni informa sobre tratamientos especializados ("nunca he necesitado tratamientos de esos, esas cosas son para gente sin carácter"). Respeto al consumo de sustancias psicoactivas, éste señala haber consumido diversas sustancias de forma esporádica desde los trece años de edad, describiendo la etiología de dicho consumo como un patrón de búsqueda de sensaciones ("he fumado porros y cocaína de vez en cuando y reconozco que alguna vez me he emborrachado, la verdad es que las drogas no me llaman la atención pero las he probado para experimentar que se siente [...] también he probado alguna vez la heroína fumada, alucinógenos y drogas de diseño [...] nunca he tenido problemas con las drogas, pero si alguna vez he querido consumir y no tenía dinero no he dudado en robarlo").

Por último, señalar que el explorado no informa sobre acontecimientos traumáticos durante su evolución psicobiográica.

\section{EXPLORACIÓN PSICOPATOLÓGICA:}

El Sr. JGV acude a la exploración practicada esposado y acompañado por dos agentes de policía, ya que se encontraba en las dependencias de los Juzgados tras ser trasladado desde su correspondiente Centro Penitenciario. Presenta un aparente buen estado de salud y nivel de higiene adecuado. Consciente, colaborador y abordable en todo momento, se encuentra correctamente orientado en tiempo, espacio y persona, presentando un estado mental dentro de la normalidad. No se detectan alteraciones amnésicas.

Comprende el motivo y alcance de la evaluación a la que es sometido, apreciándose un nivel sociocultural bajo y adecuada capacidad intelectiva según impresión clínica, con conservación plena de su capacidad de comprensión y abstracción. El juicio, entendido éste como la capacidad para evaluar una situación y actuar de forma adecuada, se encuentra conservado.

Muestra lenguaje adecuado en ritmo y contenido durante la entrevista mantenida. A nivel de la afectividad, resulta significativo que el explorado presente indiferencia afectiva y ausencia de resonancia emocional durante todo su discurso. No obstante, establece una adecuada relación con los peritos firmantes del presente desde el comienzo, aunque manifestando que no recuerda nada sobre los hechos que se le imputan. Asimismo, impresiona por mostrar una actitud seductora e incluso en ocasiones provocativa hacia los evaluadores ("a la gente le gustaría ser como yo, pero no tienen valor [...] yo no considero que lo que hacen ustedes sea trabajar, es muy cómodo estar en un despacho sentado todo el día").

Presenta curso del pensamiento intacto y contenido inalterado, sin apreciarse alteraciones sensoperceptivas ni sensomotoras durante la valoración realizada. No se evidencia sintomatología psicótica y presenta adecuado contacto con la realidad en todo momento. 
No se aprecian signos que sugieran la existencia de dificultades a nivel psicomotor, mostrándose tranquilo y sosegado durante la evaluación. No se aprecian signos de impulsividad, información coincidente con las propias referencias del peritado en las que reconoce que nunca ha hecho algo que no haya querido hacer.

El peritado no refiere sintomatología propia de sujetos drogodependientes. No se objetivan signos de dependencia ni de síndrome de abstinencia asociados al consumo de sustancias psicoactivas.

No informa sobre ideación autolítica ni en el momento actual ni durante su evolución psicobiográfica.

Por último, señalar que la exploración realizada evidencia la ausencia de otros indicadores clínicos de interés, no apreciándose indicadores disociativos ni de tipo ansioso-depresivo significativos derivados de los estresores psicosociales vigentes y evidenciándose un estado emocional eutímico, con pensamiento y afecto egosintónico.

\section{RESULTADOS DE LAS PRUEBAS ADMINISTRADAS:}

- PCL-R

A continuación se facilita una tabla resumen las distintas puntuaciones obtenidas por el peritado en la prueba.

Tabla 1. Puntuaciones obtenidas por el peritado en el PCL-R.

\begin{tabular}{|c|c|}
\hline FACTOR 1: DESAPEGO EMOCIONAL & $\begin{array}{c}\text { PUNTUACION: } \\
0=\text { NOUUZAS, } 2=\text { SI. } x=\text { OMITR }\end{array}$ \\
\hline 1. Locuacidad y encanto superficial & 2 \\
\hline 2. Egocentrismo/ Grandiosidad & 2 \\
\hline 4. Mentira patológica & 1 \\
\hline 5. Dirección/ Manipulación & 0 \\
\hline 6. Falta de remordimiento y culpabilidad & 2 \\
\hline 7. Escasa profundidad de los afectos & 2 \\
\hline 8. Insensibilidad y falta de empatia & 2 \\
\hline 16. Incapacidad para aceptar las consecuencias de sus actos & 1 \\
\hline TOTAL & 12 \\
\hline \multicolumn{2}{|l|}{ FACTOR 2: CONDUCTA ANTISOCIAL } \\
\hline 3. Necesidad de estimulación/ aburrimiento & 2 \\
\hline 9. Estilo de vida parásito & 2 \\
\hline 10. Falta de control conductual & 1 \\
\hline 12. Problemas de conducta precoces & 2 \\
\hline 13. Falta de metas realistas a largo plazo & 2 \\
\hline 14. Impulsividad & 1 \\
\hline 15. Irresponsabilidad & 2 \\
\hline 18. Delincuencia juvenil & 2 \\
\hline 19. Revocación de la libertad condicional & 0 \\
\hline TOTAL & 14 \\
\hline SUMA TOTAL DE LOS 2 FACTORES & 26 \\
\hline 11. Conducta sexual promiscua & 2 \\
\hline 17. Varias relaciones maritales breves & 0 \\
\hline 20. Versatilidad criminal & 1 \\
\hline TOTAL PUNTUACIÓN PCL-R & $29 / 40$ \\
\hline
\end{tabular}


De acuerdo a los autores de la prueba no se puede señalar que estemos ante un sujeto con personalidad psicopática propiamente dicha ya que no alcanza la puntuación de corte establecida al respecto $(\mathrm{PD}=30)$. No obstante, a modo cualitativo sí cabe destacar la presencia de numerosos indicadores y rasgos propios de los perfiles psicopáticos de personalidad, destacando tanto los relacionados con el desapego emocional del sujeto como los propios de su comportamiento antisocial (Tabla I).

\section{- MCMI-II}

De la evaluación psicométrica practicada mediante el MCMI-II, la cual resulta válida e interpretable, se desprende la existencia de un estilo de respuesta en el peritado con tendencia a ofrecer una mala imagen de sí mismo $(Z, T B=9 /)$, presentando por tanto un estilo de respuesta distorsionado acorde a la imagen mostrada durante la entrevista mantenida. Asimismo, no se objetivan alteraciones clínicas al momento de la exploración, aunque sí la presencia de significativos rasgos caracterológicos susceptibles de configurar un trastorno de la personalidad atendiendo a la evolución psicobiográfica del peritado, destacando de sobremanera los rasgos de tipo antisocial $(6 A, T B=/ / 8)$ y narcisista $(5, T B=88)$ que describen a un sujeto que presenta un patrón de desprecio hacia los derechos de los demás, con falta de empatía y necesidad de admiración [12].

\section{- MMPI-2}

De la evaluación psicométrica practicada mediante el MMPI-2, la cual resulta válida e interpretable, se desprende la existencia un estilo de respuesta con tendencia a ofrecer una mala imagen de sí mismo $(F-K=+9)$, presentando por tanto un estilo de respuesta distorsionado acorde a la imagen mostrada durante la entrevista mantenida y coincidente con el estilo de respuesta mostrado en el MCMI-II. A continuación se facilita una tabla resumen con las principales escalas de validez del MMPI-2 cumplimentado por el explorado.

Tabla 2. Escalas de validez obtenidas por el peritado en el MMPI-2.

\begin{tabular}{|l|c|c|}
\hline \multicolumn{1}{|c|}{ Escalas de validez } & P.T & Interpretación \\
\hline $\begin{array}{l}\text { Interrogante (?) } \\
\text { (No de respuestas sin contestar o contestadas como verdadero } \\
\text { y falso a la vez) }\end{array}$ & PD: 1 & Válido \\
\hline $\begin{array}{l}\text { Mentira (L) } \\
\text { (Tendencia a ofrecer una imagen favorable) }\end{array}$ & 50 & Válido \\
\hline $\begin{array}{l}\text { Incoherencia (F) } \\
\text { (Exageración de síntomas) }\end{array}$ & 70 & Probablemente válido \\
\hline $\begin{array}{l}\text { Corrección (K) } \\
\text { (Corrección, negación de síntomas) }\end{array}$ & 58 & Perfil válido \\
\hline $\begin{array}{l}\text { Fb (F Posterior) } \\
\text { (Respuestas infrecuentes o contestadas al azar) }\end{array}$ & 72 & Validez cuestionable \\
\hline VRIN (Respuestas de inconsistencia variable) & PD: 8 & Perfil válido \\
\hline TRIN (Inconsistencia de las respuestas verdadero) & PD: 9 & Perfil válido \\
\hline
\end{tabular}

No se objetivan alteraciones clínicas al momento de la exploración, aunque sí la presencia de significativos rasgos caracterológicos susceptibles de configurar un trastorno de la personalidad atendiendo a la evolución psicobiográfica del peritado, destacando sobremanera los rasgos de desviación psicopática $(P d, P T=95)$ que describen a un sujeto con un juicio pobre, inestable, irresponsable, egocéntrico, inmaduro, agresivo y con conductas antisociales [13]. 


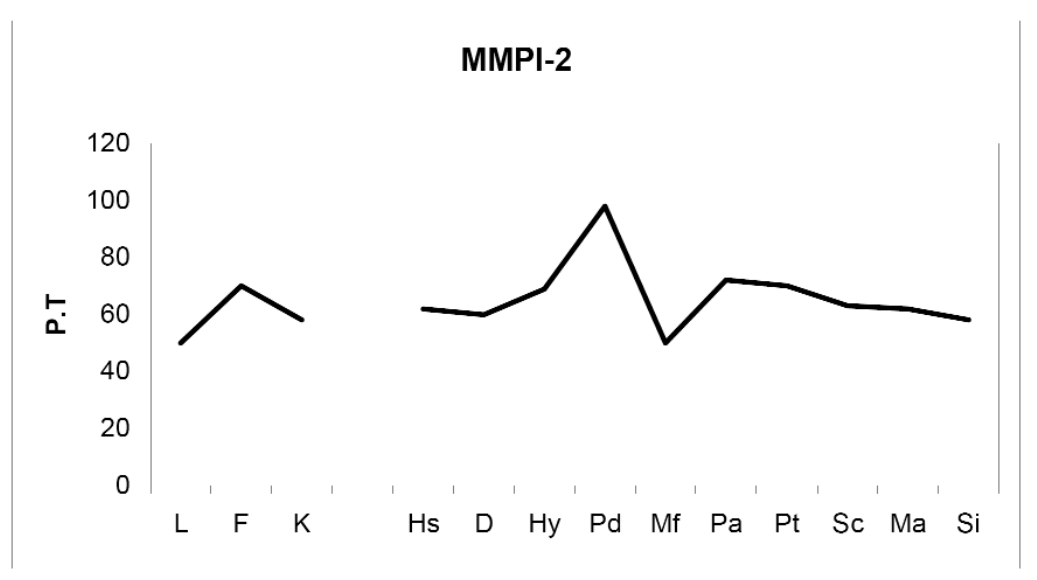

Figura 1. Perfil obtenido por el Sr. JGV en el MMPI-2.

El perfil obtenido por el peritado es propio de sujetos con marcados impulsos agresivos y hostiles, que no acierta a expresar de forma apropiada, pudiendo manifestar sentimientos muy profundos de hostilidad hacia los componentes de su familia; son personas que no aceptan las críticas y son muy sensibles al rechazo, mostrándose rebeldes y provocadores [ 14]. Siguiendo la tipología criminal de Mergagee y Bohn basada en la administración del MMPI-2 podemos señalar que el perfil obtenido corresponde al conocido como "delta", el cual se caracteriza por presentar un perfil no superior a 70 y con un pico unimodal en la escala Pd (desviación psicopática), perfil que describe a un sujeto hedonista, egocéntrico, manipulador, impulsivo y con conflictos ante la autoridad [3].

\section{DISCUSIÓN:}

Durante la evaluación practicada y atendiendo a la evolución psicobiográfica del peritado se constata una estructura de personalidad con numerosos indicadores desadaptativos de interés que configuran un F60.2 trastorno antisocial de la personalidad [30 I.7], siendo éste un trastorno con una prevalencia en la población general de varones del 3\% [9].

La característica esencial del trastorno antisocial de la personalidad es un patrón general de desprecio y violación de los derechos de los demás, que comienza en la infancia o el principio de la adolescencia y continúa en la edad adulta $[15,16]$, habiendo sido este patrón también denominado psicopatía, sociopatía o trastorno disocial de la personalidad [9]. Tal y como se ha señalado en el apartado de introducción, el concepto de psicopatía ha sido objeto de numerosas controversias y ha facilitado en ocasiones una importante confusión terminológica, de hecho, una de las fuentes de esta polémica deriva de la equiparación entre psicopático y antisocial. No obstante, debemos señalar que esta equiparación no parece del todo acertada dado que parece demostrado que ni todos los delincuentes son psicópatas, ni todos los psicópatas son delincuentes.

Para Cleckley, la falta de emociones es la característica principal del psicópata [17]. A continuación se facilita una tabla resumen con los criterios diagnósticos de psicopatía facilitados por dicho autor (Tabla 3), muchos de los cuales aparecen en el sujeto evaluado.

Resulta también de interés facilitar las nociones aportadas por Hare como continuador de la obra de Cleckley [6], señalando éste que los psicópatas se caracterizaban por la incapacidad para desarrollar relaciones afectivas con otros, la falta de empatía, la insensibilidad e indiferencia hacia los derechos y sentimientos de los demás y la ausencia de sentimientos de culpa, así como por presentar 
un estilo de vida inestable con ausencia de planes a largo plazo, una incapacidad para aceptar la responsabilidad de su conducta antisocial, la ausencia de psicopatología y por la presencia de controles conductuales débiles e inestables $[\mathrm{I} 0, \mathrm{I} \mathrm{I}]$.

Tabla 3. Criterios para el diagnóstico de psicopatía según Cleckley (1976).

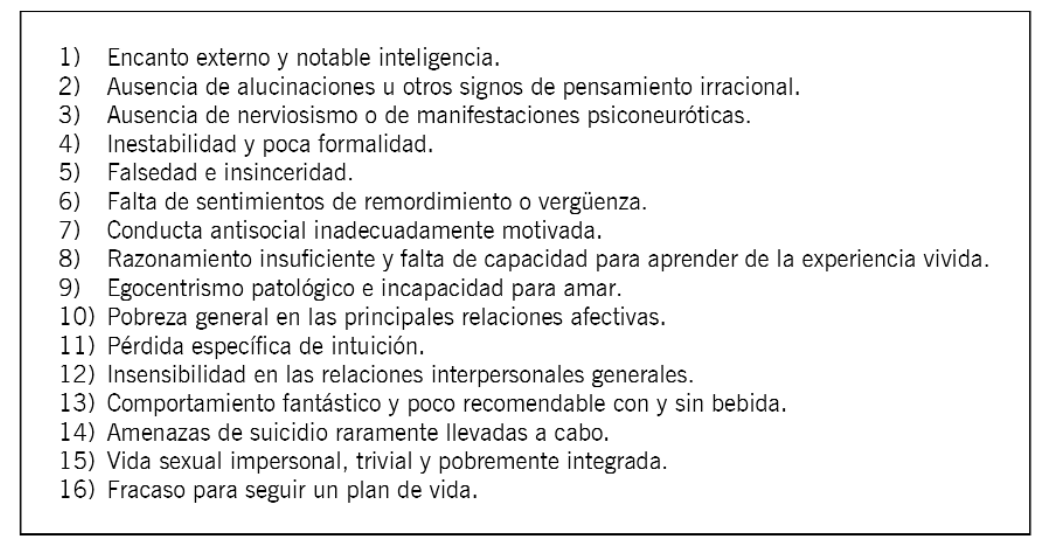

Para que se pueda establecer el diagnóstico de trastorno antisocial de la personalidad, el sujeto debe tener al menos dieciocho años y tener historia de algunos síntomas de un trastorno disocial antes de los quince años, persistiendo el comportamiento antisocial hasta la edad adulta $[9,15,16]$. Los rasgos de personalidad antisocial sólo constituyen un trastorno antisocial de la personalidad cuando son inflexibles, desadaptativos y persistentes, y ocasionan deterioro funcional significativo o malestar subjetivo [9], tal y como sucede con el Sr. JGV.

Además, los sujetos con un trastorno antisocial de la personalidad no logran adaptarse a las normas sociales en lo que respecta al comportamiento legal, pudiendo perpetrar repetidamente actos que son motivo de detención (que puede o no producirse), de hecho, los sujetos con este tipo de trastorno desprecian los deseos, derechos o sentimientos de los demás y frecuentemente, engañan y manipulan con tal de conseguir provecho o placer personales $[9,18,19]$. Son personas que pueden mentir repetidamente y presentar un patrón de impulsividad con evidente incapacidad para planificar el futuro, tal y como sucede en el presente caso. Las decisiones se toman sin pensar, sin prevenir nada y sin tener en cuenta las consecuencias para uno mismo o para los demás, lo que puede ocasionar cambios repentinos de trabajo, de lugar de residencia o de amistades [9]. Asimismo, los sujetos con un trastorno antisocial de la personalidad tienden a ser irritables y agresivos, siendo también frecuente que muestren una despreocupación imprudente por su seguridad o la de los demás, pudiendo involucrarse en comportamientos sexuales o consumo de sustancias que tengan un alto riesgo de producir consecuencias perjudiciales $[9,18,19]$. Los sujetos con trastorno antisocial de la personalidad también tienden a ser continua y extremadamente irresponsables, además de tener pocos remordimientos por las consecuencias de sus actos, llegando a ser indiferentes o dar justificaciones superficiales por haber ofendido, maltratado o robado a alguien, llegando a culpar a las víctimas por ser tontos, débiles o por merecer su mala suerte, pueden minimizar las consecuencias desagradables de sus actos $\mathrm{O}$, simplemente, mostrar una completa indiferencia $[9,15]$.

Tal y como se ha señalado, los sujetos con trastorno antisocial de la personalidad frecuentemente carecen de empatía y tienden a ser insensibles, cínicos y a menospreciar los sentimientos, derechos y penalidades de los demás, pudiendo tener un concepto de sí mismos engreído y 
arrogante, llegando a parecer excesivamente tercos, autosuficientes o fanfarrones, tal y como se constata durante la exploración psicopatológica practicada; además, pueden mostrar labia y encanto superficial y ser muy volubles y de verbo fácil $[8,9]$.

Los sujetos con trastorno antisocial de la personalidad presentan frecuentemente rasgos de personalidad de tipo límite, histriónico y narcisista; de hecho, en este caso, se ha podido apreciar la existencia de significativos rasgos caracterológicos de tipo narcisista, aunque no se cumplen los criterios diagnósticos para establecer un segundo trastorno de la personalidad. No obstante, cabe señalar que los sujetos con trastorno antisocial de la personalidad y trastorno narcisista de la personalidad comparten la tendencia a ser duros, poco sinceros, superficiales, explotadores y poco empáticos, sin embargo, el trastorno narcisista de la personalidad no incluye las características de impulsividad, agresión y engaño [ $|2$,$| | ]$, aspectos si apreciados en este caso.

Respecto al origen de este tipo de trastorno, el maltrato o el abandono en la infancia, el comportamiento inestable o variable de los padres o la inconsistencia en la disciplina por parte de los padres aumentan las probabilidades de que un trastorno disocial evolucione hasta un trastorno antisocial de la personalidad, siendo éste un trastorno que se presenta asociado a un bajo status socioeconómico y al medio urbano [9], tal y como sucede en el presente caso.

En cuanto a su curso, el trastorno antisocial de la personalidad tiene un curso crónico, pero puede hacerse menos manifiesto o remitir a medida que el sujeto se va haciendo mayor, especialmente hacia la cuarta década de la vida $[9,15]$.

Cuando el trastorno antisocial de la personalidad en un adulto está asociado a un trastorno relacionado con sustancias, no se establece el diagnóstico del trastorno antisocial de la personalidad a no ser que los signos del trastorno antisocial de la personalidad también hayan aparecido en la infancia y hayan continuado hasta la edad adulta [9], situación evidente en el presente caso, donde el trastorno de la personalidad y el abuso de sustancias se presentan de manera comórbida. De hecho, cuando el consumo de sustancias y el comportamiento antisocial hayan empezado en la infancia y continúen en la edad adulta, se diagnosticarán tanto trastorno relacionado con sustancias como trastorno antisocial de la personalidad, siempre que se cumplan los criterios para ambos trastornos, aunque algunos actos antisociales sean consecuencia del trastorno relacionado con sustancias. En este caso consideramos que el consumo de sustancias psicoactivas referido por el peritado resulta compatible con la presencia de un trastorno de abuso de sustancias, entendido éste como un patrón desadaptativo de consumo que conlleva un deterioro o malestar clínicamente significativos, expresados en el presente caso a través del consumo recurrente de sustancias, el incumplimiento de responsabilidades y los distintos problemas legales [9], todo ello acorde a su estructura de personalidad de base en la que destaca la búsqueda de sensaciones. Pese al historial de consumo de tóxicos referido por parte del peritado, al momento de la exploración no se objetivan signos o síntomas de intoxicación o de síndrome de abstinencia en relación a dichas sustancias, resultando su patrón de consumo compatible con una situación de uso/abuso de dichas sustancias, no siendo probable que el explorado haya presentado un cuadro de adicción o dependencia si atendemos a sus referencias al respecto.

Por último, debemos señalar que el trastorno descrito en el Sr. JGV describe a un sujeto que no sólo no está motivado a respetar la normativa imperante, sino que más bien está claramente enfrentado a la misma. Además, destacamos que a la hora de valorar la imputabilidad del sujeto no sólo se exige la presencia de esos rasgos, anomalías, alteraciones o trastornos de la personalidad, sino que éstos deben originar y alcanzar unos efectos determinados, tales como son la no comprensión de la ilicitud del hecho o la imposibilidad de actuar conforme a dicha comprensión. En el 
caso del explorado, debemos señalar que éste no presenta alteraciones cognitivas significativas, ni déficit intelectual, ni trastornos de la forma o el contenido del pensamiento, ni de la percepción ni de otras funciones instrumentales como la atención, concentración o memoria, siendo un sujeto que comprende y razona adecuadamente y con un sentido claro de la realidad; mientras que respecto a su capacidad volitiva, y pese a detectarse la presencia de comportamientos irresponsables e impulsivos en el peritado, éste ha demostrado ser capaz de poder controlar los mismos y llegar a planificar de forma controlada sus actos delictivos, descartándose por tanto afectación en su esfera volitiva.

Por todo ello, considerando la valoración forense realizada y tenida en cuenta en su globalidad, se debe señalar por parte de estos peritos que al momento de la exploración realizada no se detectan alteraciones psicopatológicas ni anomalías en la estructura de personalidad de base significativas en el Sr. JGV que supongan un menoscabo en su capacidad de comprender o de actuar conforme a dicha comprensión.

\section{CONCLUSIONES:}

Los peritos firmantes del presente informe han redactado su contenido con imparcialidad y con arreglo a su leal saber y entender. Con todos los respetos a SS ${ }^{\mathrm{a}} \mathrm{O}$ al Tribunal que corresponda, emiten las siguientes conclusiones:

ÚNICA: Al momento de la exploración, no se detectan alteraciones ni anomalías psicopatológicas en el Sr. JVG que supongan un menoscabo en su capacidad de comprender o de actuar conforme a dicha comprensión. Asimismo, no se objetivan durante la exploración signos o síntomas de intoxicación aguda ni de síndrome de abstinencia a sustancias psicoactivas.

Lo que informan a los efectos oportunos,

En (lugar), a (día) de (mes) de (año).

\section{BIBLIOGRAFÍA:}

I. Carrasco JJ, Maza JM. Manual de psiquiatría legal y forense. La Ley, Madrid, 2005.

2. Martínez-Garay L. La imputabilidad penal: concepto, fundamento, naturaleza jurídica y elementos. Tirant lo Blanch, Valencia, 2005. 3. Esbec E, Gómez-Jarabo G. Psicología forense y tratamiento jurídico-legal de la discapacidad. Edisofer, Madrid, 2000.

4. Consejería de Justicia e Interior de la Comunidad de Madrid. Guía orientativa de buenas prácticas de psicólogos forenses. Comunidad de Madrid, Madrid, 2007.

5. Schneider K. Las personalidades psicopáticas. Morata, Madrid, 1980.

6. López-Magro C, Robles JI. Aproximación histórica al concepto de psicopatía. Revista de Psicopatología Clínica Legal y Forense, 5, I37168, 2005.

7. Jiménez MJ, Fonseca GM. Trastornos de la personalidad (psicopatías): Tratamiento científico y jurisprudencial. CESEJ Ediciones, Madrid, 2006.

8. Organización Mundial de la Salud (OMS). Clasificación Internacional de las Enfermedades. Trastornos mentales y del comportamiento: descripciones clínicas y pautas para el diagnostico, CIE-I0. Meditor, Madrid, 1992.

9. Asociación de Psiquiatría Americana (APA). Manual diagnostico y estadístico de los trastornos mentales, DSM-IV-TR. Masson, Barcelona, 2002.

I0. Hare R. The psychopathy checklist revised. Multi-health systems, Toronto, 1991.

II. Hare R. The psychopathy checklist revised technical manual (2nd. Ed.). Multi-health systems, Toronto, 2003.

I2. Millon T. Inventario Clínico Multiaxial de Millon (MCMI-II). TEA Ediciones, Madrid, 2004.

13. Hathaway ST, McKinley JC. Inventario Multifásico de Personalidad de Minnesota-2 (MMPI-2). TEA Ediciones, Madrid, 2002.

14. Graham JR. Assesing personality and psychopathology. Oxford University Press, New York, 2000.

15. Kaplan HI, Sadock BJ. Sinopsis de psiquiatría. Editorial Panamericana, Madrid, 1999.

16. Vallejo-Ruiloba J. Introducción a la psicopatología y psiquiatría. Masson, Barcelona, 2001.

17. Cleckley H. The mask of sanity. Mosby, St. Louis, 1976.

18. Vázquez B. Manual de psicología forense. Síntesis, Madrid, 2005. 19. Soria MA. Manual de psicología jurídica e investigación criminal. Pirámide, Madrid, 2005. 